\title{
Awake Proning as an Adjunctive Therapy for Refractory Hypoxemia in Non-Intubated Patients with COVID-19 Acute Respiratory Failure: Guidance from an International Group of Healthcare Workers
}

\author{
Willemke Stilma, ${ }^{1,2 \star}$ Eva Åkerman, ${ }^{3,4}$ Antonio Artigas, ${ }^{5,6}$ Andrew Bentley, ${ }^{7,8}$ Lieuwe D. Bos, ${ }^{1}$ Thomas J. C. Bosman, ${ }^{1}$ \\ Hendrik de Bruin, ${ }^{1}$ Tobias Brummaier, ${ }^{9,10}$ Laura A. Buiteman-Kruizinga, ${ }^{1,11}$ Francesco Carcò, ${ }^{12}$ Gregg Chesney, ${ }^{13}$ Cindy Chu, ${ }^{9,10}$ \\ Paul Dark, ${ }^{14,15,16}$ Arjen M. Dondorp, ${ }^{10,17}$ Harm J. H. Gijsbers, ${ }^{18}$ Mary Ellen Gilder, ${ }^{19}$ Domenico L. Grieco, ${ }^{20,21}$ Rebecca Inglis, ${ }^{22}$ \\ John G. Laffey, ${ }^{23,24}$ Giovanni Landoni, ${ }^{12,25}$ Weihua Lu, ${ }^{26}$ Lisa M. N. Maduro, ${ }^{18}$ Rose McGready, ${ }^{9,10}$ Bairbre McNicholas, ${ }^{23}$ \\ Diego de Mendoza, ${ }^{27,28,29}$ Luis Morales-Quinteros, ${ }^{27,30}$ Francois Nosten, ${ }^{9,},{ }^{10}$ Alfred Papali, ${ }^{31,32}$ Gianluca Paternoster, ${ }^{33}$ \\ Frederique Paulus, ${ }^{1,2}$ Luigi Pisani, ${ }^{1,17,34}$ Eloi Prud'homme, ${ }^{35}$ Jean-Damien Ricard, ${ }^{36,37,38}$ Oriol Roca, ${ }^{39}$ Chiara Sartini, ${ }^{12}$ \\ Vittorio Scaravilli, ${ }^{40}$ Marcus J. Schultz, ${ }^{1,10,17}$ Chaisith Sivakorn, ${ }^{41}$ Peter E. Spronk, ${ }^{42}$ Jaques Sztajnbok, ${ }^{43}$ Youssef Trigui, ${ }^{44}$
} Kathleen M. Vollman, ${ }^{45}$ and Margaretha C. E. van der Woude ${ }^{46}$

${ }^{1}$ Department of Intensive Care, Amsterdam University Medical Centers, Location 'AMC', Amsterdam, The Netherlands; ${ }^{2}$ Faculty of Health, Center of Expertise Urban Vitality, Amsterdam University of Applied Science, Amsterdam, The Netherlands; ${ }^{3}$ Division of Nursing, Department of Neurobiology, Care Sciences and Society, Karolinska Institute, Stockholm, Sweden; ${ }^{4}$ Function of Perioperative Medicine and Intensive Care, Department of Intensive Care, Karolinska University Hospital, Stockholm, Sweden; ${ }^{5}$ Department of Intensive Care, Hospital de Sabadell, CIBER Enfermedades Respiratorias, Sabadell, Barcelona, Spain; ${ }^{6}$ Autonomous University of Barcelona, Sabadell, Barcelona, Spain; ${ }^{7}$ Acute Intensive Care Unit, Manchester University NHS Foundation, Manchester, United Kingdom; ${ }^{8}$ Manchester Academic Health Science Centre, Faculty of Biology, Medicine and Health, University of Manchester, Manchester, United Kingdom; ${ }^{9}$ Shoklo Malaria Research Unit, Mahidol-Oxford Tropical Medicine Research Unit, Faculty of Tropical Medicine, Mahidol University, Mae Sot, Thailand; ${ }^{10}$ Centre for Tropical Medicine and Global Health, Nuffield Department of Medicine, University of Oxford, Oxford, United Kingdom; ${ }^{11}$ Department of Intensive Care, Reinier de Graaf Hospital, Delft, The Netherlands; ${ }^{12}$ Department of Anesthesia and Intensive Care, IRCCS San Raffaele Scientific Institute, Milan, Italy; ${ }^{13}$ Division of Emergency Medicine-Critical Care, Department of Emergency Medicine, NYU Grossman School of Medicine, New York, New York; ${ }^{14}$ Critical Care Medicine, NIHR Manchester Biomedical Research Centre, University of Manchester, Manchester, United Kingdom; ${ }^{15}$ Division of Infection, Immunity and Respiratory Medicine, NIHR Manchester Biomedical Research Centre, University of Manchester, Manchester, United Kingdom; ${ }^{16}$ Humanitarian and Conflict Response Institute, University of Manchester, Manchester, United Kingdom; ${ }^{17}$ Faculty of Tropical Medicine, Mahidol-Oxford Tropical Medicine Research Unit (MORU), Mahidol University, Bangkok, Thailand; ${ }^{18}$ Department of Rehabilitation Medicine, Amsterdam University Medical Centers, Location 'AMC', Amsterdam, The Netherlands; ${ }^{19}$ Department of Family Medicine, Faculty of Medicine, Chiang Mai University, Chiang Mai, Thailand; ${ }^{20}$ Department of Emergency, Intensive Care Medicine and Anesthesia, Fondazione Policlinico Universitario A. Gemelli IRCCS,

Rome, Italy; ${ }^{21}$ Department of Anesthesiology and Intensive Care Medicine, Catholic University of the Sacred Heart, Rome, Italy; ${ }^{22}$ Lao-OxfordMahosot Hospital-Wellcome Trust Research Unit (LOMWRU), Mahosot Hospital, University of Oxford, Vientiane, Lao People's Democratic

Republic; ${ }^{23}$ Department of Anaesthesia and Intensive Care, MedicineGalway University Hospitals, Galway, Ireland; ${ }^{24}$ School of Medicine, Disciplines of Anaesthesia and Intensive Care Medicine, National University of Ireland, Galway, Ireland; ${ }^{25}$ School of Medicine, Vita Salute San Raffaele University, Milan, Italy; ${ }^{26}$ Department of Critical Care Medicine, Yijishan Hospital of Wannan Medical College, Wuhu, China; ${ }^{27}$ Intensive Care Department, Hospital Universitari Sagrat Cor. Grupo Quironsalud, Barcelona, Spain; ${ }^{28}$ Emergency Department, Hospital Universitari Sagrat Cor. Grupo Quironsalud, Barcelona, Spain; ${ }^{29}$ Ciber Enfermedades Respiratorias (CIBERES), Instituto de Salud Carlos III, Madrid, Spain; ${ }^{30}$ Institut d' Investigacio I Innovacio Parc Taulí I3PT, Universidad Autonoma de Barcelona, Barcelona, Spain; ${ }^{31}$ Division of Pulmonary and Critical Medicine, Atrium Health, Charlotte, North Carolina; ${ }^{32}$ School of Medicine, University of Maryland, Baltimore, Maryland; ${ }^{33}$ Department of Cardiovascular Anaesthesia and ICU, San Carlo Hospital, Potenza, Italy; ${ }^{34}$ Section of Operational Research, Doctors with Africa CUAMM, Padova, Italy; ${ }^{35}$ Intensive Care Unit, Détresse Respiratoire Infections Sévères, Assistance Publique Hôpitaux de Marseille, Marseille, France; ${ }^{36}$ DMU ESPRIT-

Enseignements et Soins de Proximité, Recherche, Innovation et Territoires, Université de Paris, Paris, France; ${ }^{37}$ Infection, Antimicrobiens, Modélisation, Evolution (IAME), Université de Paris, Paris, France; ${ }^{38}$ Service de Médecine Intensive Réanimation, Hôpital Louis Mourier, Assistance Publique - Hôpitaux de Paris, Colombes, France; ${ }^{39}$ Servei de Medicina Intensiva, Hospital Vall d'Hebron, Barcelona, Spain; ${ }^{40}$ Department of Anesthesia, Critical Care and Emergency, Fondazione IRCCS Ca' Granda - Ospedale Maggiore Policlinico, Milan, Italy; ${ }^{41}$ Department of Clinical Tropical Medicine, Mahidol University, Bangkok, Thailand; ${ }^{42}$ Expertise Center for Intensive Care Rehabilitation Apeldoorn, Gelre Hospitals Apeldoorn, Apeldoorn, The Netherlands; ${ }^{33}$ Intensive Care Unit, Instituto de Infectologia Emilio Ribas, São Paulo, Brazil; ${ }^{44}$ Service des Maladies Respiratoires, Centre Hospitalier D'Aix-en-Provence, Aix-en-Provence, France; ${ }^{45}$ Clinical Nurse Specialist/Critical Care Consultant, Advancing Nursing LLC, Northville, Michigan; ${ }^{46}$ Intensive Care Unit, Zuyderland Medisch Centrum, Location 'Heerlen', Heerlen, The Netherlands

Abstract. Non-intubated patients with acute respiratory failure due to COVID-19 could benefit from awake proning. Awake proning is an attractive intervention in settings with limited resources, as it comes with no additional costs. However, awake proning remains poorly used probably because of unfamiliarity and uncertainties regarding potential benefits and practical application. To summarize evidence for benefit and to develop a set of pragmatic recommendations for awake proning in patients with COVID-19 pneumonia, focusing on settings where resources are limited, international healthcare professionals from high and low- and middle-income countries (LMICs) with known expertise in awake proning were invited to contribute expert advice. A growing number of observational studies describe the effects of awake proning in patients with COVID-19 pneumonia in whom hypoxemia is refractory to simple measures of supplementary oxygen. Awake proning improves oxygenation in most patients, usually within minutes, and reduces dyspnea and work of breathing. The effects are maintained for up to 1 hour after turning back to supine, and mostly disappear after 6-12 hours. In available studies, awake proning was not associated with a reduction in the rate of intubation for invasive ventilation. Awake proning comes with little complications if properly implemented and monitored. Pragmatic recommendations including indications and contraindications were formulated and adjusted for resource-limited settings. Awake proning, an adjunctive treatment for hypoxemia refractory to supplemental oxygen, seems safe in non-intubated patients with COVID-19 acute respiratory failure. We provide pragmatic recommendations including indications and contraindications for the use of awake proning in LMICs.

\footnotetext{
${ }^{*}$ Address correspondence to Willemke Stilma, Department of Intensive Care, Amsterdam University Medical Centers, Location 'AMC', Meibergdreef 9, Amsterdam 1105 AZ, The Netherlands.
} E-mail: w.stilma@amsterdamumc.nl 


\section{INTRODUCTION}

COVID-19 acute respiratory failure may cause severe hypoxemia. ${ }^{1}$ Many patients need to be hospitalized for supplementary oxygen. If this fails, that is, when hypoxemia is refractory to oxygen therapy, invasive ventilation is often needed.

In intubated and invasively ventilated patients with moderate-to-severe acute respiratory distress syndrome, prone positioning can improve oxygenation and has been shown to improve survival. ${ }^{2,3}$ Benefit of prone positioning may not be restricted to invasively ventilated patients-at least in theory, non-intubated patients could also benefit from being placed in a prone position. ${ }^{4,5}$ The so-called awake proning is a cheap intervention, and thus very attractive from an economic viewpoint. Awake proning, however, could be or become uncomfortable if incorrectly performed, especially when it needs to be provided for many hours. It may also come with complications such as shoulder injuries, ${ }^{6,7}$ pressure ulcers, ${ }^{8}$ and aspiration of gastric content. ${ }^{9}$

We invited a group of healthcare professionals with known interest or expertise in awake proning or with practical knowledge regarding care for patients with acute respiratory failure in low- and middle-income countries (LMICs), to develop a set of pragmatic recommendations for use of this intervention. The goal was to develop a guidance enriched with illustrations for a better understanding and local training of healthcare professionals. Information on awake proning mainly originated in resource-rich settings in high-income countries-the group translated the available information into recommendations for use in resource-restricted settings in LMICs.

\section{METHODS}

An international group of healthcare professionals was invited by the study leads (W. S., L. D. B., L. P., M. J. S., and F. P.). Communication and writing within the group and three subgroups was merely by email correspondence and teleconferences, and a central shared online document was used to draft the current guidance.

Several literature searches in Medline were performed, using different combinations of search terms like "coronavirus disease," "coronavirus disease 2019," "COVID-19," "SARSCoV-2," "prone positioning" and "awake proning," "nonintubated," "non-invasive," "oxygen therapy," "high flow nasal oxygen (HFNO)," "non-invasive ventilation (NIV)," "respiratory monitoring," and "ratio of oxygen saturation (ROX) index." Searches had to be updated several times, as publications continued to appear in the literature during the writing of this report. A final search in Medline was performed in late October 2020. In addition to these Medline searches, Internet searches, mainly through the Google search machine and using the comparable terms, were performed to explore the gray literature and search for webinars on awake proning.

Information was bundled and dealt within three subgroups regarding the following questions: 1) What is the evidence for benefit of awake proning for acute respiratory failure in general, and in COVID-19 pneumonia in particular? 2) What are the recommendations and suggestions for practical application of awake proning for acute respiratory failure in general, and in COVID-19 pneumonia? 3) Should recommendations for use of awake proning differ between high-income countries and
LMICs? Members could participate in more than one subgroup, by members' preferences.

Quality of evidence was scored from very high to very low, and the strength of each recommendation was given as strong or weak considering indirectness of evidence and magnitude of effects. For LMICs, the availability, affordability, safety, and feasibility of awake proning in patients with acute respiratory failure were used to refine the recommendations if necessary. ${ }^{10}$

\section{RESULTS}

Reports from the subgroups: Evidence for benefit. The number of studies investigating awake proning is rapidly increasing, but thus far, randomized clinical trials remain absent. Published studies were heterogeneous with regard to several aspects-for example, supplementary oxygen during awake proning was provided using diverse interfaces, from simple oxygen supplementation via a nasal prong or cannula ${ }^{11-14}$ or a Venturi mask $^{14}$ to continuous positive airway pressure (CPAP), ${ }^{15-18} \mathrm{HFNO},{ }^{4,12,19,20}$ and $\mathrm{NIV}^{21}$; the exact positions taken during awake proning also differed widely; thresholds for awake proning varied, from pulse oximetry as high as $>94 \%{ }^{15,22}$ to as low as $<90 \%{ }^{11}$; duration of awake proning varied too, from 30 minutes to several hours $4,5,11,15,21,23$ or even longer ${ }^{13,19,20,22,24}$; and proning could be applied more times per day, ${ }^{4,5,21}$ or until low oxygen saturations resolved. ${ }^{14}$

Awake proning improves oxygenation ${ }^{4,5,11,12,15,19,25}$ and also reduces dyspnea. ${ }^{13,21,22}$ The improvements in oxygenation are seen within minutes after the start of awake proning. ${ }^{11}$ The effects of awake proning on oxygenation are maintained for up to one hour after turning back to supine ${ }^{15}$ but disappear after 6-12 hours. ${ }^{12,26}$ Awake proning also reduces dyspnea sensation and work of breathing by improving ventilation-perfusion. ${ }^{14,19,21,22,25}$ Despite this benefit, awake proning is not always tolerated. ${ }^{5,12,14,21,27}$

It remains uncertain whether awake proning can prevent invasive ventilation. Several studies show a low intubation rate with the use of awake proning. ${ }^{12,14,21}$ Two studies suggest prevention of intubation, ${ }^{20,22}$ but this is not confirmed in other investigations in invasively ventilated patients, ${ }^{15,28,29}$ nor in patients with severe hypoxemia receiving NIV, ${ }^{4}$ nor in patients receiving $\mathrm{HFNO}^{30}$ It is highly uncertain whether awake proning can be used as a rescue therapy, that is, to avoid intubation in patients who already fulfill the criteria for immediate intubation.

It remains unclear whether the effects of awake proning depend on the way supplemental oxygen is administered, albeit that improvements in oxygenation are described with all forms of oxygen supplementation, that is, via nasal cannula or oxygen mask, ${ }^{11-13,15,19,28,31}$ CPAP, ${ }^{15,21} \mathrm{HFNO}^{12,20,24,27,30,31}$ and NIV. ${ }^{4,21}$ Some studies suggest that "early" awake proning (i.e., when oxygen can still be supplied via a simple interface like a nasal cannula) ${ }^{11,12,22,28}$ could have a better effect than "late" awake proning (i.e., when oxygen needs to be supplied via HFNO or CPAP). ${ }^{15,21,24,32}$ A change in pulse oximetry readings or respiratory rate $(R R)$ induced by awake proning could be useful parameters to define responders versus nonresponders. In one study, a rise of $\mathrm{SpO}_{2}>95 \%$ is associated with a lower intubation rate. ${ }^{28}$

Persistent hypoxemia despite supplementary oxygen was used as an indication in all studies, albeit with considerable variation in the degree of hypoxia. Literature remains vague 
regarding other indications, and also contraindications for awake proning. Based on the studies identified by the searches and consensus within the group, indications and (relative) contraindications for awake proning were formulated (Table 1). Consensus was not based on robust evidence, and may depend on various factors such as available resources, and local expertise-for example, in a hospital ward environment with a low nurse-to-patient ratio, it may be challenging to safely use light sedation in the management of a severely dyspneic patient who will receive awake proning.

Reports from the subgroups: Practical application of awake proning. Based on the studies identified by the searches and consensus within the group, recommendations and suggestions for practical application of awake proning in COVID19 patients were formulated (Table 2). The group considers it essential to train local teams in terms of correct and safe use of awake proning, especially when light sedation is used. Ideally, an "awake proning team" consists of two healthcare professionals, including at least one doctor, and a nurse or a physical therapist. One professional should provide team leadership. The team will assist the patient to take the correct position and ascertains continuation of oxygenation supplementation-extra oxygen during positioning could be considered. The healthcare professional should assist in proper positioning of the limbs. Supporting materials, such as pillow blocks, cushions, or rolled blankets, should be closely at hand. To minimize the risks of awake proning, a practical checklist or "proning bundle" can be checked every time a patient is placed in the prone position ${ }^{33}$ (Table 3).

For awake proning to be successful, the group thinks motivational support to the patient is one key to success. Before proning, why awake proning could work, what it is like to be in a prone position, and how to maintain a proper position should be explained to the patient and family members if present. A potential increase on pulse oximetry readings and a reduction in dyspnea, coughing, and sputum production could increase following the position change-this is usually short-lasting. It can be useful to know if the patient normally sleeps face down (e.g., prone), to adjust this information. Patients could find awake proning uncomfortable, but this may be mitigated through supportive nursing care and noticing the marked improvements of pulse oximetry readings. Family can stay with patients who are proning, and use of mobile devices to spend time and communicate with relatives should be stimulated. If use of sedatives or anxiolytics is being considered to facilitate prone positioning in non-intubated patients, this should be undertaken in a closely monitored location, with access to continuous oxygen saturation, blood pressure, and electrocardiogram monitoring. Pain medication could be considered, as pain related to stiffness of shoulders and neck could develop.

Frequent assessment for tolerability, at least within 10 minutes after the start of proning, is considered important. In some patients, it may be necessary to start benzodiazepine, clonidine, or dexmedetomidine, but only if the setting allows; also, morphine in low dosages could be useful in a severely dyspneic patient. In those cases, proper monitoring, including continuous or intermittent pulse oximetry, blood pressure, and maybe electrocardiogram could be useful.

The vast majority of patients will receive supplemental oxygen through interfaces like a nasal prong or cannula, a face mask, CPAP via a mask, or a ventilation hood, HFNO, or NIV. ${ }^{15-17,19}$ There is no evidence as to which interface is best in patients who receive prone positioning. Nasal interfaces and masks seem more practical and better tolerated than hoods, ${ }^{5}$ especially in elderly patients. ${ }^{15}$ One current multicenter randomized clinical trial is testing the efficacy of different

TABLE 1

Indications and contraindications to apply awake proning

Indications

$\mathrm{SpO}_{2} / \mathrm{FiO}_{2}$ ratio $<315$

Acute respiratory failure requiring any supplemental oxygen to maintain saturation $>90 \%$

Able to follow instructions in their native language

Absolute contraindications in the ward and ICU setting

Anticipated difficult airway

Cardiogenic pulmonary edema as a cause for respiratory failure

Respiratory rate of above $40 /$ min or accessory muscle use

Unreliable $\mathrm{SpO}_{2}$ tracing

Immobile or extremely limited mobility

Inability to tolerate proning due to anatomic concerns (e.g., injury or wound on the ventral surface of the body)

Spinal instability

Glaucoma or other condition with acutely elevated intraocular pressure

Severe head trauma with high ICP

Absolute contraindications in the ward, but relative contraindication in the ICU setting

Severe oxygenation problems defined as $\mathrm{PaO}_{2} / \mathrm{FiO}_{2}<100 \mathrm{mmHg}$ or, alternatively, $\mathrm{SpO}_{2} / \mathrm{FiO}_{2}<140 \mathrm{mmHg}$

Altered mental status or inability to follow commands

Inability to communicate with care team or call for help verbally or with call bell

Hemodynamic instability defined as requiring vasopressor support (i.e., a systolic blood pressure $<90 \mathrm{mmHg}$ or mean arterial pressure less than $65 \mathrm{mmHg}$ despite appropriate volume resuscitation)

Inability to reposition self for comfort without assistance

Relative contraindications in the ward and ICU setting

Facial injury

Neurological issues (e.g., frequent seizures)

Morbid obesity $(\mathrm{BMI}>40)$

Pregnancy (2/3rd trimesters)

Pressure ulcers

Concomitant type II respiratory failure, unless chronic, stable, and compensated $(\mathrm{pH}>7.36)$. If awake proning is considered, it should be trialed, and a blood gas should be taken within 30 minutes to ensure no deterioration in hypercapnia. 
TABLE 2

Recommendations and suggestions for practical application of awake proning in COVID-19 patients (with grading)

\begin{tabular}{|c|c|c|}
\hline & Domain & Recommendation \\
\hline 1 & Indications & $\begin{array}{l}\text { Suggest: Consider awake proning in } \\
\text { patients with acute respiratory failure } \\
\text { requiring supplemental oxygen } \\
\text { to maintain saturation }>93 \% .^{11,15,22}\end{array}$ \\
\hline 2 & Indications & $\begin{array}{l}\text { Suggest: Consider awake proning in } \\
\text { patients able to follow instructions. }\end{array}$ \\
\hline 3 & Indications & $\begin{array}{l}\text { Recommend: Use awake proning during } \\
\text { the } 1 \text { st and } 2 \text { nd trimesters in pregnant } \\
\text { women with additional monitoring of } \\
\text { the position and the fetus. }\end{array}$ \\
\hline 4 & Contra-indications & $\begin{array}{l}\text { Suggest: Use awake proning in the 3rd } \\
\text { trimester of pregnancy with additional } \\
\text { monitoring with caution and on an } \\
\text { individual risk-benefit basis. }\end{array}$ \\
\hline 5 & Contra-indications & $\begin{array}{l}\text { Recommend against: Awake proning in } \\
\text { patients with extreme respiratory } \\
\text { distress requiring immediate } \\
\text { intubation. }{ }^{15,20,22,28,29}\end{array}$ \\
\hline 6 & Contra-indications & $\begin{array}{l}\text { Suggest against: Awake proning in } \\
\text { patients with impaired consciousness. }\end{array}$ \\
\hline 7 & Preparation & $\begin{array}{l}\text { Strongly recommend: Preparing the } \\
\text { patient and the family for what it is like to } \\
\text { be in a prone position, what can be } \\
\text { expected, and how to maintain this } \\
\text { position. }\end{array}$ \\
\hline 8 & Preparation & $\begin{array}{l}\text { Recommend: Preparation for } \\
\text { complications (safe airway, suctioning, } \\
\text { and pressure ulcers). }\end{array}$ \\
\hline 9 & Monitoring & $\begin{array}{l}\text { Strongly recommend: Minimum } \\
\text { monitoring of pulse rate and peripheral } \\
\text { oxygen saturation. }\end{array}$ \\
\hline
\end{tabular}

Expert opinion

$\begin{array}{ll}\text { Low-quality evidence } & \begin{array}{c}\text { Where pulse oximetry is not available, it } \\ \text { would be reasonable to trial awake } \\ \text { proning for COVID-19 patients with } \\ \text { cyanosis, marked tachypnea, or other } \\ \text { evidence of respiratory distress. }\end{array} \\ \text { Expert opinion } & \text { No additional considerations. }\end{array}$

Expert opinion

In settings without tocography and Doppler, fetal monitoring using clinical auscultation of the fetal heart rate should be performed.

Expert opinion

In settings without tocography and Doppler, fetal monitoring using clinical auscultation of the fetal heart rate should be performed.

Low-quality evidence

Where mechanical ventilation is not available or affordable, a trial of awake proning may be performed as a rescue maneuver.

Low-quality evidence

No additional considerations.

Expert opinion

Visual aids may be useful to illustrate the family what will happen. Caregivers will often become a key component of the proning team.

Widely available fleece blankets can be used instead of pillows to reduce costs.

Expert opinion

Examples of recommended preparations for complications include having the equipment necessary for emergency intubation prepared nearby in case it is required, having a functioning suction machine with a clean suction catheter available at all times, and ensuring careful padding of all pressure areas and daily pressure area surveillance.

Where available, a multiparametric monitor or a handheld or tabletop pulse oximeter is preferable to a fingertip pulse oximeter (not easily seen or heard from a distance and may automatically switch off after a certain time period). ${ }^{61}$ Whatever device is used, the alarm should be set to alert staff if $\mathrm{SpO}_{2}$ drops below $90 \%$.

When there are insufficient pulse oximeters available for continuous monitoring, intermittent monitoring should be carried out as frequently as staffing and equipment allow.

10 Monitoring

11 Monitoring

12 Monitoring

13 Monitoring

14 Monitoring

15 Oxygen supply
Recommend: Monitoring respiratory rate, work of breathing (use of respiratory muscles), and dyspnea.

Suggest: Possibility of monitoring respiratory status by using the ROX index.

Recommend: Monitoring of hemodynamic parameters (MAP and SBP).

Suggest: Visual care monitoring by open wards in event of high surge capacity.

Suggest against: Awake proning in conventional hospital wards for patients with severe respiratory failure.

Recommend: Use of any available method of oxygen delivery during awake proning.
Expert opinion

Expert opinion

Expert opinion

Expert opinion

Expert opinion

Expert opinion
While safety is high, feasibility depends on the local level of staffing. ${ }^{62}$

Feasibility relies on the availability of pulse oximetry.

We recommend a noninvasive blood pressure measurement at least once an hour where possible (expert opinion).

This is a pragmatic measure that improves patient safety and makes efficient use of staff and PPE.

This recommendation may not apply in settings where no higher level of care is available.

Oxygen is a scarce resource in at least one-quarter of hospitals in LMICs. ${ }^{52}$ The choice among oxygen concentrators, cylinders, or centralized 

affordable option. Attention should be paid to ensuring the tubing is not kinked in the prone position and in the case of a reservoir mask that the bag is fully inflated.

16 Oxygen supply

17 Position

18 Position

19 Position

20 Position

21 Position

22 Position

23 Position

24 Hydration and nutrition

25 Hydration and nutrition

26 Hydration and nutrition

27 Risk management

28 Risk management

29 Risk management

30 Risk management
Suggest: Use of CPAP or HFNO for delivery of higher $\mathrm{FiO}_{2}$, depending on the locally available expertise.

Suggest: Train multidisciplinary proning teams in approaches on awake and sedated proning with one person having the lead.

Suggest: Have a slightly lateral position to turn the face.

Suggest: Avoid a closed packed shoulder by keeping the shoulder of the raised arm around $80^{\circ}$ abduction. ${ }^{35}$

Suggest: Full flexion of the knees if possible and maximum range ankle motion.

Suggest: Use analgesia when low back pain becomes a problem.

Recommend: Supportive padding above and below the gravid uterus when pregnant women are proned (Figure 1).

Suggest: A semi-lateral prone position in pregnant woman in the $2 \mathrm{nd} / 3 \mathrm{rd}$ trimester as an alternative (Figure 1).

Recommend: Maintain normovolemia.

Suggest: Allow oral intake unless there is a high risk of intubation.

Suggest: Stay in the supine position for one hour after oral feeding in the supine position.

Recommend: Have equipment for endotracheal intubation nearby and frequently checked.

Recommend: Have an intravenous port available for sudden clinical deterioration.

Recommend: Have materials for (endotracheal or nasal) suctioning standby.

Suggest: Start reverse CPR until a team is ready to get the patient in the supine position.
Low-quality evidence

Expert opinion

Expert opinion

Expert opinion

Expert opinion

Expert opinion

Expert opinion

Expert opinion

Expert opinion

Expert opinion

Expert opinion

Expert opinion

Expert opinion

Expert opinion

Expert opinion

Availability and affordability of CPAP and HFNO systems is variable but generally low. ${ }^{64}$ Feasibility of HFNO is low because of high oxygen demands. Where insufficient staff are available, care givers can also provide support. ${ }^{65}$

Some patients prefer to keep their head central rather than turned to the side (see Figure 2 for a configuration of padding to accommodate this).

No additional considerations.

Extra pillows may be needed. Widely available fleece blankets can be used instead of pillows to reduce costs.

Folded fleece blankets can be used for this purpose.

No additional recommendations.

No additional recommendations. No additional recommendations.

No additional recommendations.

This only applies to centers where mechanical ventilation is available.

No additional recommendations.

Where electrical suction devices are not available, a manual suction pump or bulb suction can be used.

CPR should only be commenced once staff attending the patient are wearing N95 respirators/masks or equivalent.

$\mathrm{CPAP}=$ continuous positive airway pressure; $\mathrm{CPR}=$ cardiopulmonary resuscitation; $\mathrm{HFNO}=$ high-flow nasal oxygen; $\mathrm{LMICs}=$ low- and middle-income countries; $\mathrm{MAP}=$ mean arterial pressure; $\mathrm{NIV}=$ noninvasive ventilation; $\mathrm{PPE}=$ personal protective equipment; $\mathrm{SBP}=$ systolic blood pressure

${ }^{\star}$ Considerations regarding feasibility, availability, safety and affordibility..$^{10}$

interfaces for supplemental oxygen during awake proning in patients with COVID-19 acute respiratory failure. ${ }^{34}$

In patients with mild hypoxemia, "self-proning" could be possible, eventually with the help of a family member. In patients with severe hypoxemia, "assisted proning" is likely to be superior to "self-proning," as patients may need all of their energy to breathe, and thus need help. Assistance also prevents dislocation of the interfaces for supplemental oxygen and any indwelling catheter. Assistance may prevent an increase in oxygen consumption induced by changing the position, especially in older, frail, pregnant, and obese patients.
Some refinements could increase safety of awake proning and may allow acceptance for a longer period of time. Suggested positions are illustrated in Figures 1 and 2 . A slightly lateral prone position allows a patient to turn the face to one side, which can be supported by a pillow or rolled blanket placed under one side of the chest, and a raised arm embracing the pillow (the "front crawl" or "swimmers position"). While in the prone position, the patient faces the armpit of the raised arm of which the elbow is flexed at $\sim 90^{\circ}$, and the contralateral arm remains aligned with the body. A maximum closed packed position of the shoulder is avoided by keeping the shoulder of the raised arm at $\sim 80^{\circ}$ abduction, or even lower 
TABLE 3

Safe awake proning checklist

\begin{tabular}{|c|c|c|}
\hline Preparation & Proning & After turning/during proning \\
\hline Patient & Patient & Patient \\
\hline Identity & Self-proning & Comfort \\
\hline Explanation procedure & Assisted proning & $\begin{array}{l}\text { Document chosen position (prone and } \\
\text { lateral) }\end{array}$ \\
\hline $\begin{array}{l}\text { Document duration of procedure } \\
\text { Consent }\end{array}$ & & Document position of arms \\
\hline Materials & Materials & Materials \\
\hline Pillows and slide sheet & $\begin{array}{l}\text { Sufficient room between the head and } \\
\text { shoulders for oxygen supply }\end{array}$ & $\begin{array}{l}\text { Provide emergency buzzer, mobile } \\
\text { phone, and improvised rattle }\end{array}$ \\
\hline Crash cart & $\begin{array}{l}\text { In pregnant women, special attention to } \\
\text { alleviate pressure on the gravid uterus }\end{array}$ & \\
\hline \multicolumn{3}{|l|}{ Oxygen available } \\
\hline \multicolumn{3}{|l|}{ Suction equipment available } \\
\hline \multicolumn{3}{|l|}{ Monitoring: pulse oximetry if available } \\
\hline Check & & Check \\
\hline Vital signs: $\mathrm{SpO}_{2}, \mathrm{RR}, \mathrm{HR}$, and $\mathrm{BP}$ & Oxygen supply continued & Vital signs: $\mathrm{SpO}_{2}, \mathrm{RR}, \mathrm{HR}$, and $\mathrm{BP}$ \\
\hline IV access & & IV access \\
\hline Nurse call system & & Nurse call system \\
\hline \multirow[t]{6}{*}{ Baby monitor in case of pregnancy } & & Additional external fetal monitoring \\
\hline & & Medication \\
\hline & & Pain: paracetamol 4 dd $1 \mathrm{~g}$ \\
\hline & & Anxiety: low-dose benzodiazepine \\
\hline & & Oxazepam 10 mg po \\
\hline & & Midazolam 1-2 mg po \\
\hline Emergencies & Emergencies & Emergencies \\
\hline Emergency team for the supine position & Emergency team for the supine position & Emergency team for the supine position \\
\hline Crash cart (intubation equipment) & Crash cart (intubation equipment) & Crash cart (intubation equipment) \\
\hline
\end{tabular}

if possible. ${ }^{35}$ The upper arm and shoulder blade are positioned in a straight horizontal line to protect the shoulder, and arm repositioning is encouraged, if pain or stiffness occurs. Slight adjustments or repositioning of the legs and hip should be encouraged to prevent pressure ulcers and meralgia paresthetica. The latter complication is a result of compression injury of the lateral femoral cutaneous nerve. ${ }^{36}$ The side of the swimmers position changes frequently, preferably every 2 or 3 hours. Full flexion of the knees should be possible, with a maximum range of ankle motion to prevent stiffness and pointed feet. In patients with lower back pain, a semi-prone or lateral position could be taken. If this does not provide relief for the lower back pain, pain medication could be considered.

As patients in prone position may need urgent intubation, they should remain fasting. Fasting is also advisable because of an increased risk for aspiration when in a prone position. Patients should thus be encouraged to take oral feeds in supine, head-up position, between the sessions, if allowed, and should not be placed in a prone position for at least 1 hour after oral intake. Fluids should be given intravenously, if needed. However, sips of water could be taken with the bed in a more upright position.

Direct visual care and monitoring of patients during awake proning is facilitated by designated areas for cohorts of patients. Monitoring of RR, accessory muscle use, and work of breathing could help identifying patients who need escalation of care. The ROX index, ${ }^{37}$ defined as the ratio of $\mathrm{SpO}_{2} / \mathrm{FiO}_{2}$ to $\mathrm{RR}$, has been proposed for monitoring. This index combines three parameters that assess a patient's respiratory status. Improvement in the ROX index during awake proning could indicate a lesser likelihood for intubation, ${ }^{38}$ but experience with the ROX index in patients with COVID-19 acute respiratory failure is still very limited.

In case of cardiac arrest in a prone position, "reverse cardiopulmonary resuscitation (CPR)"39,40 can and must be
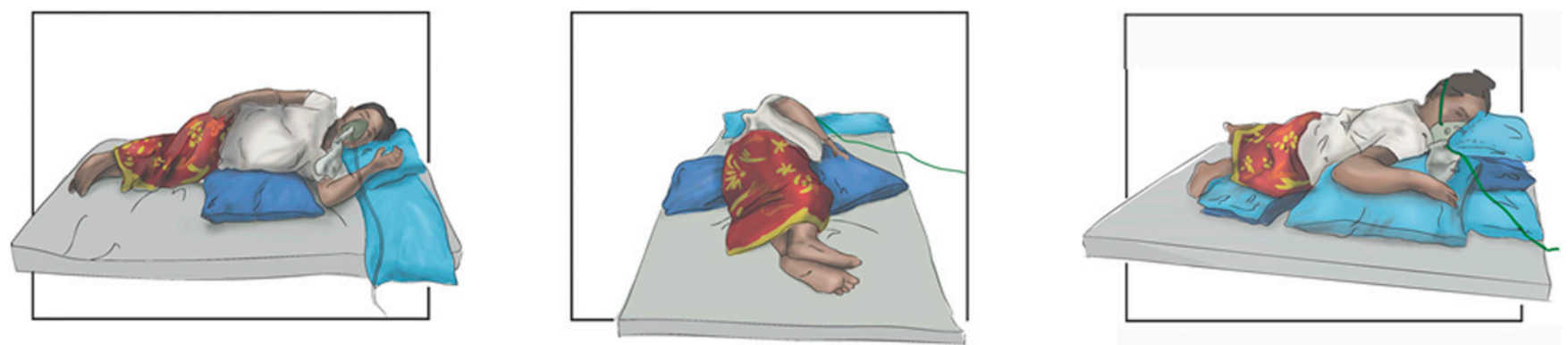

FIGURE 1. Awake proning in a 9-month pregnant woman. Both $3 / 4$ prone and full prone options are shown. Suggested position is an indication and could be adapted based on patient preferences. This figure appears in color at www.ajtmh.org. 


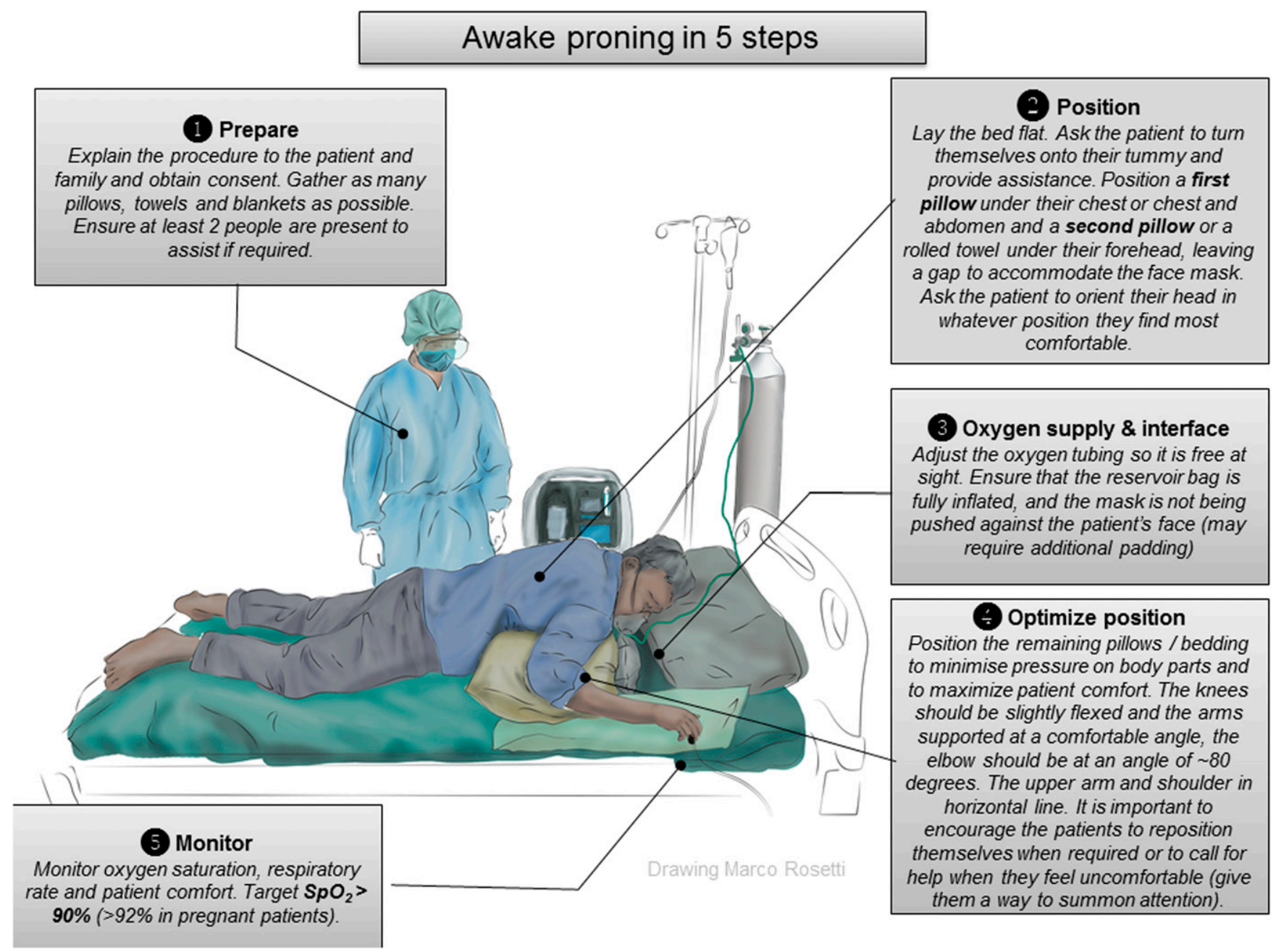

FIGURE 2. Visual aid to facilitate awake proning implementation in a resource-limited setting. Suggested position is an indication and could be adapted based on patient preferences. Adapted with permission from a prone positioning checklist developed by Dr. Rebecca Inglis in Lao PDR. ${ }^{67}$ This figure appears in color at www.ajtmh.org.

started until a team is available to turn the patient back to supine. ${ }^{41}$ This team should be identified and always be readily available. Emergency endotracheal intubation equipment and materials must be close by and regularly checked for immediate use alongside relevant emergency drugs.

Finally, the group suggests awake proning should not be withheld in pregnant patients, ${ }^{42-47}$ but supplemental oxygen should be provided such that pulse oximetry remains $\geq 95 \%$. ${ }^{43}$ To prevent aortocaval compression in pregnant patients, ${ }^{48}$ additional measures can be taken by organizing extra pillows and monitoring.

Reports from the subgroups: Recommendations for awake proning in LMICs. As awake proning does not require particular resources, it should be considered in hypoxemic patients with COVID-19 acute respiratory failure who do not respond to simple supplementary oxygen in settings where resources are limited. The group considers awake proning with any available method of respiratory support a safe intervention, also in LMICs. Awake proning may prevent the need for invasive ventilation which is important for settings with limited numbers of ventilators. ${ }^{14,32}$ In fact, at times, awake proning may be the only option to improve oxygenation in settings. Limitations regarding awake proning in LMICs include a lack of human resources, training, and challenges with infrastructure and equipment. ${ }^{49}$

The group recognizes the limitation of staffing in many LMICs and that a "proning team" may not always be feasible. The guidance by one trained healthcare professional, or two in an obese or a noncooperative patient, however, is seen as one minimal requirement to proceed with this intervention. It is always important that a patient can be turned back to supine with urgency to allow emergency procedures such as CPR. Training of staff becomes pivotal in limited-resource settings, as it is possible to spare time and resources when exact maneuvers are known by the team. Figures 1 and 2 provide additional training material.

Oxygen is listed as an essential medication by the $\mathrm{WHO}^{50}$ but remains a very limited resource in many settings. ${ }^{51-53}$ Low-flow supplemental oxygen via nasal prongs, spongetipped catheters, or face masks are increasingly available and affordable, although one-quarter of hospitals surveyed in an LMIC study reported gaps in oxygen supply. ${ }^{52}$ Facemasks with reservoir allow increasing $\mathrm{FiO}_{2}$ significantly and should be strongly considered (Figure 2). High-flow nasal oxygen and NIV are feasible in LMICs, but not widely available. They come at additional costs for the interfaces and devices. They also 
come with technical challenges and practical concerns, as they depend on a reliable source of oxygen and electricity. Continuous positive airway pressure and HFNO apparatuses may rapidly use up oxygen supplies; indeed, HFNO consumes oxygen at more than four times the rate of low flow oxygen support, and typically, HFNO may consume the entire content of a large oxygen cylinder within 2-3 hours, rapidly depleting oxygen supply.

Close monitoring and clear escalation criteria are needed with awake proning, also in resource-limited settings. In LMICs, pulse oximeters are often not available, but recent initiatives have been set up to provide them on a larger scale. ${ }^{54}$ Pulse oximetry together with monitoring of clinical and vital signs might help timely identification of those patients who need escalation of care. ${ }^{55}$ In settings where blood gas analyzers are unavailable, $\mathrm{SpO}_{2}$ relative to inspiratory oxygen concentration, or $\mathrm{SpO}_{2} / \mathrm{FiO}_{2}$ ratio, can be used for continuous monitoring, decision-making, and prognostication. ${ }^{56-58}$ The ROX index is likely to be useful because it requires simple input $\left(\mathrm{SpO}_{2} /\right.$ $\mathrm{FiO}_{2}$ and respiratory rate) and is easy to calculate at the bedside. ${ }^{59}$

During the supine periods between awake proning, oral intake is to be encouraged to maintain normovolemia in resource-limited settings, as other resources for fluid intake are usually limited. The risk of aspiration, however, should be highlighted, especially in obese patients. The suggestion of using low-dose benzodiazepines or morphine to enhance awake proning should be conducted with care in environments with limited or absent patient monitoring.

\section{DISCUSSION}

A rapidly growing number of observational studies describe the use of awake proning in patients with COVID-19 acute respiratory failure in whom hypoxemia is refractory to simple supplementary oxygen. Awake proning improves oxygenation within minutes, and the effects are maintained for up to one hour after turning back to supine and disappear mostly after 6-12 hours. Awake proning is associated with few complications. Because no particular technological resources are required, it is particularly applicable in settings where resources are limited, or even absent. A set of pragmatic recommendations were formulated on awake proning in relation to indications and contraindications, oxygen supply, position, nutrition, monitoring, and risk management based on the available evidence and experiences of healthcare workers in LMICs.

It is important to notice that so far there is no randomized trial evidence for the effect of awake proning in patients with COVID-19 acute respiratory failure. Current evidence comes from few studies, mostly case reports and single-center observations. None of these originated in LMICs. The available results, however, suggest that awake proning could be effective adjunctive therapy that is also safe and tolerable. The suggestion that it may prevent the need for invasive ventilation or increased need of oxygen makes this intervention worth a try, certainly in settings where there is a shortage of ventilators or where ventilators are absent ${ }^{20,22}$ or health care is unaffordable for patients.

One strength of this guidance on awake proning is the cooperation of a set of healthcare workers from resourcerich settings in high-income countries and from resourcelimited settings in LMICs. There was a large expertise in proning, and a deep understanding of the challenges in intensive care units in LMICs. Also, the group consisted of various types of healthcare workers, including (ICU) doctors, (ICU) nurses, and physiotherapists.

This work also has limitations. We emphasize that this is not a systematic review, but rather a clinical appraisal of the available literature and personal clinical experiences of healthcare workers in various settings around the world. We cannot exclude selection and information bias.

\section{CONCLUSION}

Awake proning is an attractive and safe adjunctive treatment for hypoxemia refractory to supplemental oxygen in patients with COVID-19 acute respiratory failure, especially in settings where there is shortage or absolute lack of ventilators. Here, this could be the only option to improve oxygenation. It may even prevent the need for invasive ventilation; although randomized trial evidence remains lacking, randomized clinical trials are urgently needed.

Received November 9, 2020. Accepted for publication February 28, 2021.

Published online March 11, 2021.

Acknowledgments: The American Society of Tropical Medicine and Hygiene has waived the Open Access fee for this article due to the ongoing COVID-19 pandemic.

Financial support: This study received funding from the Amsterdam University Medical Center, location "AMC"; Center of Expertise Urban Vitality, Faculty of Health, Amsterdam University of Applied Science.

Disclosure: All authors contributed to the drafting of this manuscript. Paul Dark was supported by the NIHR Manchester Biomedical Research Centre. For the purpose of open access, the author has applied a CC BY public copyright license to any author-accepted manuscript version arising from this submission. This research was funded in whole, or in part, by the Wellcome Trust [Grant number 220211] (authors T. B., C. C., R. McG., and F. N.).

\section{SUBGROUP CHAIRS AND MEMBERS}

Group 1: Evidence for benefit of awake proning

Chairs: Willemke Stilma and Marcus J. Schultz; members (in alphabetic order): Andrew Bentley, Hendrik de Bruin, Gregg Chesney, Domenico L. Grieco, Giovanni Landoni, Diego de Mendoza, Luis Morales-Quinteros, Gianluca Paternoster, Oriol Roca, Chiara Sartini, Vittorio Scaravilli, Chaisit Sivakorn, Peter E. Spronk, Jaques Sztajnbok, and Youssef Trigui.

Group 2: Practical application of awake proning

Chairs: Frederique Paulus and Lieuwe D. Bos; members (in alphabetic order): Eva Åkerman, Thomas J.C. Bosman, Laura A. BuitemanKruizinga, Gregg Chesney, Cindy Chu, Paul Dark, Harm J.H. Gijsbers, Weihua Lu, Lisa M.N. Maduro, Bairbre McNicholas, Eloi Prud'homme, and Margaretha C.E. van der Woude.

Group 3: Specific recommendations for use of awake proning in LMICs

Chairs: Luigi Pisani and Marcus J. Schultz; members (in alphabetic order): Eva Åkerman, Antonio Artigas, Andrew Bentley, Tobias Brummaier, Francesco Carcò, Cindy Chu, Arjen M. Dondorp, Mary Ellen Gilder, Rebecca Inglis, John G. Laffey, Rose McGready, François Nosten, Gianluca Paternoster, Alfred Papali, Jean-Damien Ricard, Oriol Roca, Chaisith Sivakorn, Peter E. Spronk, and Kathleen M. Vollman.

Authors' addresses: Willemke Stilma and Frederique Paulus, Department of Intensive Care, Amsterdam University Medical Centers, Location 'AMC', Amsterdam, The Netherlands, and Faculty of Health, Center of Expertise Urban Vitality, Amsterdam University of 
Applied Science, Amsterdam, The Netherlands, E-mail: w.stilma@ amsterdamumc.nl and f.paulus@amsterdamumc.nl. Eva Åkerman, Division of Nursing, Department of Neurobiology, Care Sciences and Society, Karolinska Institute, Stockholm, Sweden, and Function of Perioperative Medicine and Intensive Care, Department of Intensive Care, Karolinska University Hospital, Stockholm, Sweden, E-mail: eva.akerman@gmail.com. Antonio Artigas, Department of Intensive Care, Hospital de Sabadell, CIBER Enfermedades Respiratorias, Sabadell, Barcelona, Spain, and Autonomous University of Barcelona, Sabadell, Barcelona, Spain, E-mail: aartigas@tauli.cat. Andrew Bentley, Acute Intensive Care Unit, Manchester University NHS Foundation, Manchester, United Kingdom, and Faculty of Biology, Medicine and Health, Manchester Academic Health Science Centre, University of Manchester, Manchester, United Kingdom, E-mail: andrew.bentley@manchester.ac.uk. Lieuwe D. Bos, Thomas J. C. Bosman, and Hendrik de Bruin, Department of Intensive Care, Amsterdam University Medical Centers, Location 'AMC', Amsterdam, The Netherlands, E-mails: I.d.bos@amsterdamumc.nl, t.j.bosman@ amsterdamumc.nl, and h.debruin2@amsterdamumc.nl. Tobias Brummaier, Cindy Chu, Rose McGready, Francois Nosten, Shoklo Malaria Research Unit, Mahidol-Oxford Tropical Medicine Research Unit, Faculty of Tropical Medicine, Mahidol University, Mae Sot, Thailand, and Centre for Tropical Medicine and Global Health, Nuffield Department of Medicine, University of Oxford, Oxford, United Kingdom, E-mails: tobias.brummaier@gmx.at, cindy@tropmedres.ac, rose@shoklo-unit.com, and francois@tropmedres.ac. Laura A. Buiteman-Kruizinga, Department of Intensive Care, Amsterdam University Medical Centers, Location 'AMC', Amsterdam, The Netherlands, and Department of Intensive Care, Reinier de Graaf Hospital, Delft, The Netherlands, E-mail: I.kruizinga@rdgg.nl. Francesco Carcò, Department of Anesthesia and Intensive Care, IRCCS San Raffaele Scientific Institute, Milan, Italy, E-mail: carco.fn@gmail.com. Gregg Chesney, Division of Emergency Medicine-Critical Care, Department of Emergency Medicine, NYU Grossman School of Medicine, New York, NY, E-mail: gregg.chesney@nyulangone.org. Paul Dark, Critical Care Medicine, NIHR Manchester Biomedical Research Centre, University of Manchester, Manchester, United Kingdom, Division of Infection, Immunity and Respiratory Medicine, NIHR Manchester Biomedical Research Centre, University of Manchester, Manchester, United Kingdom, and Humanitarian and Conflict Response Institute, University of Manchester, Manchester, United Kingdom, E-mail: paul.m.dark@manchester.ac.uk. Arjen M. Dondorp, Faculty of Tropical Medicine, Mahidol-Oxford Tropical Medicine Research Unit (MORU), Mahidol University, Bangkok, Thailand, E-mail: arjen@ tropmedres.ac. Harm J. H. Gijsbers and Lisa M. N. Maduro, Department of Rehabilitation Medicine, Amsterdam University Medical Centers, Location 'AMC', Amsterdam, The Netherlands, E-mails: h.j.gijsbers@amsterdamumc.nl and I.m.maduro@amsterdamumc.nl. Mary Ellen Gilder, Department of Family Medicine, Faculty of Medicine, Chiang Mai University, Chiang Mai, Thailand, E-mail: mellietyros@gmail.com. Domenico L. Grieco, Department of Emergency, Intensive Care Medicine and Anesthesia, Fondazione Policlinico Universitario A. Gemelli IRCCS, Rome, Italy, and Department of Anesthesiology and Intensive Care Medicine, Catholic University of the Sacred Heart, Rome, Italy, E-mail: dlgrieco@outlook.it. Rebecca Inglis, Lao-Oxford-Mahosot Hospital-Wellcome Trust Research Unit (LOMWRU), Mahosot Hospital, University of Oxford, Vientiane, Lao People's Democratic Republic, E-mail: rebecca.i@tropmedres.ac. John G. Laffey, Department of Anaesthesia and Intensive Care, MedicineGalway University Hospitals, Galway, Ireland, and School of Medicine, Disciplines of Anaesthesia and Intensive Care Medicine, National University of Ireland, Galway, Ireland, E-mail: john.laffey@ nuigalway.ie. Giovanni Landoni, Department of Anesthesia and Intensive Care, IRCCS San Raffaele Scientific Institute, Milan, Italy, and School of Medicine, Vita Salute San Raffaele University, Milan, Italy, E-mail: landoni.giovanni@hsr.it. Weihua Lu, Department of Critical Care Medicine, Yijishan Hospital of Wannan Medical College, Wuhu, China, E-mail: Iwh683@126.com. Bairbre McNicholas, Department of Anaesthesia and Intensive Care, MedicineGalway University Hospitals, Galway, Ireland, E-mail: bairbre.nimhaille@hse.ie. Diego de Mendoza, Intensive Care Department, Hospital Universitari Sagrat Cor. Grupo Quironsalud, Barcelona, Spain, Emergency Department, Hospital Universitari Sagrat Cor. Grupo Quironsalud, Barcelona, Spain, and Ciber Enfermedades Respiratorias (CIBERES), Instituto de Salud Carlos III, Madrid, Spain, E-mail: diego.mendoza@ quironsalud.es. Luis Morales-Quinteros, Intensive Care Department, Hospital Universitari Sagrat Cor. Grupo Quironsalud, BarceIona, Spain, and Institut d' Investigacio I Innovacio Parc Taulí I3PT, Universidad Autonoma de Barcelona, Barcelona, Spain, E-mail: luchomq2077@gmail.com. Alfred Papali, Division of Pulmonary and Critical Medicine, Atrium Health, Charlotte, NC, and School of Medicine, University of Maryland, Baltimore, MD, E-mail: alfred. papali@atriumhealth.org. Gianluca Paternoster, Department of Cardiovascular Anaesthesia and ICU, San Carlo Hospital, Potenza, Italy, E-mail: paternostergianluca@gmail.com. Luigi Pisani, Department of Intensive Care, Amsterdam University Medical Centers, Location 'AMC', Amsterdam, The Netherlands, Faculty of Tropical Medicine, Mahidol-Oxford Tropical Medicine Research Unit (MORU), Mahidol University, Bangkok, Thailand, and Section of Operational Research, Doctors with Africa CUAMM, Padova, Italy, E-mail: luigipisani@ gmail.com. Eloi Prud'homme, Intensive Care Unit, Détresse Respiratoire Infections Sévères, Assistance Publique Hôpitaux de Marseille, Marseille, France, E-mail: eloiprudhomme@gmail.com. Jean-Damien Ricard, DMU ESPRIT-Enseignements et Soins de Proximité, Recherche, Innovation et Territoires, Université de Paris, Paris, France, Infection, Antimicrobiens, Modélisation, Evolution (IAME), Université de Paris, Paris, France, and Service de Médecine Intensive Réanimation, Hôpital Louis Mourier, Assistance Publique - Hôpitaux de Paris, Colombes, France, E-mail: jean-damien.ricard@aphp.fr. Oriol Roca, Servei de Medicina Intensiva, Hospital Vall d'Hebron, Barcelona, Spain, E-mail: oroca@vhebron.net. Chiara Sartini, Department of Anesthesia and Intensive Care, IRCCS San Raffaele Scientific Institute, Milan, Italy, E-mail: doc.chiara.sartini@gmail.com. Vittorio Scaravilli, Department of Anesthesia, Critical Care and Emergency, Fondazione IRCCS Ca' Granda - Ospedale Maggiore Policlinico, Milan, Italy, E-mail: vittorio.scaravilli@gmail.com. Marcus J. Schultz, Department of Intensive Care, Amsterdam University Medical Centers, Location 'AMC', Amsterdam, The Netherlands, Centre for Tropical Medicine and Global Health, Nuffield Department of Medicine, University of Oxford, Oxford, United Kingdom, and Faculty of Tropical Medicine, Mahidol-Oxford Tropical Medicine Research Unit (MORU), Mahidol University, Bangkok, Thailand, E-mail: marcus.j.schultz@gmail.com. Chaisith Sivakorn, Department of Clinical Tropical Medicine, Mahidol University, Bangkok, Thailand, E-mail: chaisith.siv@mahidol.edu. Peter E. Spronk, Expertise Center for Intensive Care Rehabilitation Apeldoorn, Gelre Hospitals Apeldoorn, Apeldoorn, The Netherlands, E-mail: p.spronk@gelre.nl. Jaques Sztajnbok, Intensive Care Unit, Instituto de Infectologia Emilio Ribas, São Paulo, Brazil, E-mail: jaques.sztajnbok@hc.fm.usp.br. Youssef Trigui, Service des Maladies Respiratoires, Centre Hospitalier D'Aixen-Provence, Aix-en-Provence, France, E-mail: youssef.trigui@ gmail.com. Kathleen M. Vollman, Clinical Nurse Specialist/Critical Care Consultant, Advancing Nursing LLC, Northville, MI, E-mail: kvollman@comcast.net. Margaretha C. E. van der Woude, Intensive Care Unit, Zuyderland Medisch Centrum, Location 'Heerlen', Heerlen, The Netherlands, E-mail: m.vanderwoude@zuyderland.nl.

This is an open-access article distributed under the terms of the Creative Commons Attribution (CC-BY) License, which permits unrestricted use, distribution, and reproduction in any medium, provided the original author and source are credited.

\section{REFERENCES}

1. Wiersinga WJ, Prescott HC, 2020. What is COVID-19? JAMA 324: 816.

2. Ayzac L, Girard R, Baboi L, Beuret P, Rabilloud M, Richard JC, Guérin C, 2016. Ventilator-associated pneumonia in ARDS patients: the impact of prone positioning. A secondary analysis of the PROSEVA trial. Intensive Care Med 42: 871-878.

3. Bloomfield R, Noble DW, Sudlow A, 2015. Prone position for acute respiratory failure in adults. Cochrane Database Syst Rev 2015: Cd008095.

4. Ding L, Wang L, Ma W, He H, 2020. Efficacy and safety of early prone positioning combined with HFNC or NIV in moderate to severe ARDS: a multi-center prospective cohort study. Crit Care 24: 28.

5. Scaravilli V, Grasselli G, Castagna L, Zanella A, Isgrò S, Lucchini A, Patroniti N, Bellani G, Pesenti A, 2015. Prone positioning improves oxygenation in spontaneously breathing nonintubated 
patients with hypoxemic acute respiratory failure: a retrospective study. J Crit Care 30: 1390-1394.

6. Kibler WB, Ludewig PM, McClure PW, Michener LA, Bak K, Sciascia AD, 2013. Clinical implications of scapular dyskinesis in shoulder injury: the 2013 consensus statement from the 'Scapular Summit'. Br J Sports Med 47: 877-885.

7. McQuade KJ, Borstad J, de Oliveira AS, 2016. Critical and theoretical perspective on scapular stabilization: what does it really mean, and are we on the right track? Phys Ther 96: 1162-1169.

8. Mora-Arteaga JA, Bernal-Ramírez OJ, Rodríguez SJ, 2015. The effects of prone position ventilation in patients with acute respiratory distress syndrome. A systematic review and metaanalysis. Med Intensiva 39: 359-372.

9. Lucchini A et al., 2017. Enteral nutrition during prone positioning in mechanically ventilated patients. Assist Inferm Ric 36: 76-83.

10. Schultz MJ, Dunser MW, Dondorp AM, 2019. Development of the guidelines: focus on availability, feasibility, affordability, and safety of interventions in resource-limited settings. Dondorp AM, Dunser MW, Schultz MJ, eds. Sepsis Management in Resource-Limited Settings. Cham, Switserland: Springer International Publishing, 25-30.

11. Caputo ND, Strayer RJ, Levitan R, 2020. Early self-proning in awake, non-intubated patients in the emergency department: a single ED's experience during the COVID-19 pandemic. Acad Emerg Med 27: 375-378.

12. Elharrar X, Trigui Y, Dols AM, Touchon F, Martinez S, Prud'homme E, Papazian L, 2020. Use of prone positioning in nonintubated patients with COVID-19 and hypoxemic acute respiratory failure. JAMA 323: 2336-2338.

13. Elkattawy S, Noori M, 2020. A case of improved oxygenation in SARS-CoV-2 positive patient on nasal cannula undergoing prone positioning. Respir Med Case Rep 30: 101070.

14. Ng Z, Tay WC, Ho CHB, 2020. Awake prone positioning for nonintubated oxygen dependent COVID-19 pneumonia patients. Eur Respir J 56: 2001198.

15. Coppo A et al., 2020. Feasibility and physiological effects of prone positioning in non-intubated patients with acute respiratory failure due to COVID-19 (PRON-COVID): a prospective cohort study. Lancet Respir Med 8: 765-774.

16. Winearls S, Swingwood EL, Hardaker CL, Smith AM, Easton FM, Millington KJ, Hall RS, Smith A, Curtis KJ, 2020. Early conscious prone positioning in patients with COVID-19 receiving continuous positive airway pressure: a retrospective analysis. BMJ Open Respir Res 7: e000711.

17. Paternoster G, Sartini C, Pennacchio E, Lisanti F, Landoni G, Cabrini L, 2020. Awake pronation with helmet continuous positive airway pressure for COVID-19 acute respiratory distress syndrome patients outside the ICU: a case series. Med Intensiva 44: 1-7. doi: 10.1016/j.medin.2020.08.008.

18. Ramirez GA et al., 2020. Continuous positive airway pressure and pronation outside the intensive care unit in COVID 19 ARDS. Minerva Med. doi: 10.23736/S0026-4806.20.06952-9.

19. Despres C, Brunin Y, Berthier F, Pili-Floury S, Besch G, 2020. Prone positioning combined with high-flow nasal or conventional oxygen therapy in severe COVID-19 patients. Crit Care 24: 256.

20. Xu Q, Wang T, Qin X, Jie Y, Zha L, Lu W, 2020. Early awake prone position combined with high-flow nasal oxygen therapy in severe COVID-19: a case series. Crit Care 24: 250.

21. Sartini $C$, Tresoldi $M$, Scarpellini $P$, Tettamanti $A$, Carco F, Landoni G, Zangrillo A, 2020. Respiratory parameters in patients with COVID-19 after using noninvasive ventilation in the prone position outside the intensive care unit. JAMA 323: 2338-2340.

22. Sztajnbok J, Maselli-Schoueri JH, Cunha de Resende Brasil LM, Farias de Sousa L, Cordeiro CM, Sansao Borges LM, Malaque $\mathrm{C}, 2020$. Prone positioning to improve oxygenation and relieve respiratory symptoms in awake, spontaneously breathing nonintubated patients with COVID-19 pneumonia. Respir Med Case Rep 30: 101096.

23. Bamford P, Bently A, Dean J, Whitmore D, Wilson-Baig N, 2020. ICS Guidance for Prone Positioning of the Conscious COVID Patient. London, United Kingdom: Intensive care society. Available at: https://icmanaesthesiacovid-19.org/news/icsguidance-for-prone-positioning-of-the-conscious-covidpatient-2020. Accessed November 1, 2020.
24. Slessarev M, Cheng J, Ondrejicka M, Arntfield R, 2020. Patient self-proning with high-flow nasal cannula improves oxygenation in COVID-19 pneumonia. Can J Anaesth 67: 1288-1290.

25. Moghadam VD, Shafiee H, Ghorbani M, Heidarifar R, 2020. Prone positioning in management of COVID-19 hospitalized patients. Braz J Anesthesiol 70: 188-190.

26. Hallifax RJ et al., 2020. Successful awake proning is associated with improved clinical outcomes in patients with COVID-19: single-centre high-dependency unit experience. BMJ Open Respir Res 7: e000678.

27. Perez-Nieto OR, Guerrero-Gutierrez MA, Deloya-Tomas E, Namendys-Silva SA, 2020. Prone positioning combined with high-flow nasal cannula in severe noninfectious ARDS. Crit Care 24: 114.

28. Thompson AE, Ranard BL, Wei Y, Jelic S, 2020. Prone positioning in awake, nonintubated patients with COVID-19 hypoxemic respiratory failure. JAMA Intern Med 180: 1537-1539.

29. Ferrando $C$ et al., 2020. Clinical features, ventilatory management, and outcome of ARDS caused by COVID-19 are similar to other causes of ARDS. Intensive Care Med 46: 2200-2211.

30. Ferrando $\mathrm{C}$ et al., 2020. Awake prone positioning does not reduce the risk of intubation in COVID-19 treated with high-flow nasal oxygen therapy: a multicenter, adjusted cohort study. Crit Care 24: 597.

31. Damarla M, Zaeh S, Niedermeyer S, Merck S, Niranjan-Azadi A, Broderick B, Punjabi N, 2020. Prone positioning of nonintubated patients with COVID-19. Am J Respir Crit Care Med. 202: 604-606.

32. Paul V, Patel S, Royse M, Odish M, Malhotra A, Koenig S, 2020. Proning in non-intubated (PINI) in times of COVID-19: case series and a review. J Intensive Care Med 35: 818-824.

33. Baldi M, Sehgal IS, Dhooria S, Agarwal R 2017. Prone positioning?: remember ABCDEFG. Chest 151: 1184-1185.

34. Perkins GD et al., 2020. RECOVERY- respiratory support: respiratory strategies for patients with suspected or proven COVID-19 respiratory failure; continuous positive airway pressure, high-flow nasal oxygen, and standard care: a structured summary of a study protocol for a randomised controlled trial. Trials 21: 687 .

35. Oliviera VM et al., 2016. Good practices for prone positioning at the bedside: construction of a care protocol. Rev Assoc Med Bras 62: 287-293.

36. Bellinghausen AL, LaBuzetta JN, Chu F, Novelli F, Rodelo AR, Owens RL, 2020. Lessons from an ICU recovery clinic: two cases of meralgia paresthetica after prone positioning to treat COVID-19-associated ARDS and modification of unit practices. Crit Care 24: 580.

37. Roca O, Caralt B, Messika J, Samper M, Sztrymf B, Hernandez G, Garcia-de-Acilu M, Frat JP, Masclans JR, Ricard JD, 2019. An index combining respiratory rate and oxygenation to predict outcome of nasal high-flow therapy. Am J Respir Crit Care Med 199: 1368-1376.

38. Zucman N, Mullaert J, Roux D, Roca O, Ricard JD, 2020. Prediction of outcome of nasal high flow use during COVID-19related acute hypoxemic respiratory failure. Intensive Care Med 46: 1924-1926.

39. Mazer SP, Weisfeldt M, Bai D, Cardinale C, Arora R, Ma C, Sciacca RR, Chong D, Rabbani LE, 2003. Reverse CPR: a pilot study of CPR in the prone position. Resuscitation 57: 279-285.

40. Moscarelli A, lozzo P, Ippolito M, Catalisano G, Gregoretti C, Giarratano A, Baldi E, Cortegiani A, 2020. Cardiopulmonary resuscitation in prone position: a scoping review. Am J Emerg Med 38: 2416-2424.

41. Barker J, Koeckerling D, West R, 2020. A need for prone position CPR guidance for intubated and non-intubated patients during the COVID-19 pandemic. Resuscitation 151: 135-136.

42. Oxford-Horrey C, Savage M, Prabhu M, Abramovitz S, Griffin K, LaFond E, Riley L, Easter SR, 2020. Putting it all together: clinical considerations in the care of critically ill obstetric patients with COVID-19. Am J Perinatol 37: 1044-1051.

43. Tolcher MC, McKinney JR, Eppes CS, Muigai D, Shamshirsaz A, Guntupalli KK, Nates JL, 2020. Prone positioning for pregnant women with hypoxemia due to coronavirus disease 2019 (COVID-19). Obstet Gynecol 136: 259-261. 
44. Oliveira C, Lopes MAB, Rodrigues AS, Zugaib M, Francisco RPV, 2017. Influence of the prone position on a stretcher for pregnant women on maternal and fetal hemodynamic parameters and comfort in pregnancy. Clinics (Sao Paulo) 72: 325-332.

45. Dennis AT, Hardy L, Leeton L, 2018. The prone position in healthy pregnant women and in women with preeclampsia - a pilot study. BMC Pregnancy Childbirth 18: 445.

46. Vibert F, Kretz M, Thuet V, Barthel F, De Marcillac F, Deruelle P, Lecointre L, 2020. Prone positioning and high-flow oxygen improved respiratory function in a 25 -week pregnant woman with COVID-19. Eur J Obstet Gynecol Reprod Biol 250: 257-258.

47. Samanta S, Samanta S, Wig J, Baronia AK, 2014. How safe is the prone position in acute respiratory distress syndrome at late pregnancy? Am J Emerg Med 32: 687 e681-683.

48. Trikha A, Ray B, 2018. Prone position ventilation in pregnancy: concerns and evidence. J Obstet Anaesth Crit Care 8: 7-9.

49. Inglis R, Ayebale E, Schultz MJ, 2019. Optimizing respiratory management in resource-limited settings. Curr Opin Crit Care 25: 45-53.

50. World Health Organisation Expert Committee on the Selection Use of essential Medicines, 2011 WHO Model List of Essential Medicines: 17th List, March 2011. Available at: https:// apps.who.int/iris/handle/10665/70640. Accessed November 1, 2020.

51. Belle J, Cohen H, Shindo N, Lim M, Velazquez-Berumen A, Ndihokubwayo JB, Cherian M, 2010. Influenza preparedness in low-resource settings: a look at oxygen delivery in 12 African countries. J Infect Dev Ctries 4: 419-424.

52. Meara JG et al., 2015. Global surgery 2030: evidence and solutions for achieving health, welfare, and economic development. Lancet 386: 569-624.

53. Papali A, Verceles AC, Augustin ME, Colas LN, Jean-Francois $\mathrm{CH}$, Patel DM, Todd NW, McCurdy MT, West TE, Haiti RLICSG, 2017. Sepsis in Haiti: prevalence, treatment, and outcomes in a Port-au-Prince referral hospital. J Crit Care 38: 35-40.

54. Lifebox, "\#MyLifesavingOximeter Helps Me to Fight COVID-19". Available at: https://www.lifebox.org/my-lifesaving-oximeterchristian-masudi/. Accessed November 1, 2020.

55. Enoch AJ, English M, Clinical Information N, McGivern G, Shepperd S, 2019. Variability in the use of pulse oximeters with children in Kenyan hospitals: a mixed-methods analysis. PLOS Med 16: e1002987.
56. Pisani Let al., 2017. Risk stratification using SpO2/FiO2 and PEEP at initial ARDS diagnosis and after $24 \mathrm{~h}$ in patients with moderate or severe ARDS. Ann Intensive Care 7: 108.

57. Riviello ED, Kiviri W, Twagirumugabe T, Mueller A, BannerGoodspeed VM, Officer L, Novack V, Mutumwinka M, Talmor DS, Fowler RA, 2016. Hospital incidence and outcomes of the acute respiratory distress syndrome using the Kigali modification of the Berlin definition. Am J Respir Crit Care Med 193: 52-59.

58. Serpa Neto A, Cardoso SO, Ong DS, Esposito DC, Pereira VG, Manetta JA, Slooter AJ, Cremer OL, 2013. The use of the pulse oximetric saturation/fraction of inspired oxygen ratio for risk stratification of patients with severe sepsis and septic shock. J Crit Care 28: 681-686.

59. Hill NS, Ruthazer R, 2019. Predicting outcomes of high-flow nasal cannula for acute respiratory distress syndrome. An index that ROX. Am J Respir Crit Care Med 199: 1300-1302.

60. Bower $\mathrm{G}, \mathrm{He} \mathrm{H}, 2020$. Protocol for awake prone positioning in COVID-19 patients: to do it earlier, easier, and longer. Crit Care 24: 371.

61. Starr $\mathrm{N}$ et al., 2020. Pulse oximetry in low-resource settings during the COVID-19 pandemic. Lancet Glob Health 8: e1121-e1122.

62. Folke M, Cernerud L, Ekstrom M, Hok B, 2003. Critical review of non-invasive respiratory monitoring in medical care. Med Biol Eng Comput 41: 377-383.

63. Howie SR et al., 2009. Meeting oxygen needs in Africa: an options analysis from the Gambia. Bull World Health Organ 87: 763-771.

64. Patel SP, Pena ME, Babcock Cl, 2015. Cost-effectiveness of noninvasive ventilation for chronic obstructive pulmonary disease-related respiratory failure in Indian hospitals without ICU facilities. Lung India 32: 549-556.

65. Papali A, Schultz MJ, Dunser MW, 2018. Recommendations on infrastructure and organization of adult ICUs in resourcelimited settings. Intensive Care Med 44: 1133-1137.

66. Oliveira VM, Piekala DM, Deponti GN, Batista DCR, Minossi SD, Chiste M, Bairros PMN, Naue WDS, Welter DI, Vieira SRR, 2017. Safe prone checklist: construction and implementation of a tool for performing the prone maneuver. Rev Bras Ter Intensiva 29: 131-141.

67. Essential Critical Care, 2020. COVID-19 Resources. Available at: https://www.essentialcriticalcare.org. Accessed February 26, 2021. 